\title{
Using gamification to break barriers to adherence in physical therapy
}

\author{
Iyad Al-Nasri, Shahan Salim
}

\begin{abstract}
Adherence to physical therapy can have a significant positive impact on the outcome of treatment, but remains a challenge for both clinicians and patients. Ubiquitous and accessible technologies such as video games and virtual reality (VR) can help to increase adherence in physical therapy, but adoption of these technologies is a barrier in itself. As such, health care professionals must play a larger role in game and simulation design in order for these interventions to be used in removing barriers to adherence.
\end{abstract}

\section{INTRODUCTION}

Adherence to a recommended treatment is an important factor that can significantly influence its outcome and success. Defined as "the extent to which a person's behavior...corresponds with agreed recommendations from a healthcare provider", adherence has shown more desirable outcomes than non-adherence. ${ }^{1}$ However, adherence to physical therapy exercises remains one of the biggest challenges in the field of rehabilitation science. ${ }^{1,2}$ From a patient perspective, physiotherapy is complicated, frustrating, and tedious, and generally comes with both monetary and time constraints. ${ }^{3-6}$ Compounding this is the fact that rehabilitation science has yet to advance sufficiently to enable clinicians to monitor patients during their day-to-day routines.

Ubiquitous and consumer-accessible technologies can allow clinicians to address both of these problems. In particular, combining video games with physiotherapy aids in making physiotherapy both intrinsically motivating and accessible without adding a significant burden to healthcare costs. " "Gamification" is defined as the use of game design elements in nongame contexts. ${ }^{8}$ The idea is to use elements from games in a vast range of real-life contexts to deal with real issues, as these components can be an effective medium to engage and motivate users. ${ }^{9}$ The purpose of this feature article is to describe two issues with physical therapy adherence: (1) there are many barriers that patients face that lead to non-adherence to physical therapy; (2) there are many barriers to adopting new innovative technologies (eg gamification) in physical therapy that could otherwise encourage intrinsic motivation for adherence in patients.

\section{Issue 1: Barriers in Treatment}

Preliminary research suggests patient non-adherence can be as high as $70 \%,{ }^{1,2}$ which states that the majority of patients do not adhere to the recommended treatment. In physiotherapy, the concept of adherence is multidimensional and relates to many different factors such as attendance at appointments, following advice, undertaking prescribed exercise programs, frequency of undertaking these exercises, correct performance of exercises, and doing more or less than advised. ${ }^{10}$ Non-adherence in physical therapy remains one of the biggest challenges in the field. ${ }^{1}$

The extent of adherence in physiotherapy remains unclear as it has a biopsychosocial component that is not often addressed during consultation. Patients face a multitude of barriers in adhering to their physiotherapy treatments. A literature review by Gardiner et al showed that the following are impediments to treatment adherence: low levels of physical activity prior to physiotherapy, low levels of adherence in the course of treatment, low selfefficacy, depression, anxiety, helplessness, poor social support, increased pain levels, and a greater perceived number of barriers. ${ }^{1}$ Furthermore, physiotherapy as a treatment option has its own barriers. Physiotherapy is not entirely covered by both Medicare and Medicaid in the United States or provincial/territorial coverage in Canada, as there are limitations on the number of visits and the total costs of the treatment expenses. ${ }^{11}$ There are also lengthy wait times for publicly-funded physiotherapy in both the United States and Canada. In-clinic treatments can ensure adherence, but they come at a significant cost to the patients themselves or to an already strained healthcare system. Lastly, an ideal clinician-patient relationship is a symbiotic partnership where the patient assumes control of their own treatment based on the recommendations made by the clinician. However, misinterpretation of instructions, complex treatment regimens, and frustration with the complexity of these regimens exacerbates the issue of non-adherence in physiotherapy.

Non-adherence is a problem that has significant implications for both the outcome and success of treatment, and it results in greater healthcare costs. If adherence can be improved with better clinic-based treatment, can we simulate that treatment, environment, and even the physiotherapist themselves in patients' homes without increasing the burden on the healthcare system?

\section{Issue 2: Failure to Adopt Innovative Technologies}

Technology has rapidly advanced in the past decade, particularly in the fields of robotics, bionics and virtual reality (VR). In many areas of healthcare, these advances have been embraced. For example, some medical schools now utilize VR based simulators that allow future doctors to experience real life scenarios in a simulated environment, resulting in better preparedness with real life cases. ${ }^{12}$ Furthermore, simulated 3-dimensional reconstruction of organs from radiological cross sections is being used as a diagnostic tool by providing clinicians with a naturalistic view of the patient 
anatomy. ${ }^{13}$ Physiotherapy as a field, unlike other professions in healthcare, has been slow to adopt new technologies. It is difficult for clinicians to embrace these ubiquitous technologies due to the lack of customization available, as the nature of rehabilitation requires hands-on applications. Control of parameters such as speed, duration, and difficulty of an intervention are paramount to clinicians. However, it is the engineers and computer scientists that have been at the forefront of producing technologies that improve patient engagement; as a result, rehabilitative technologies are often developed without the input of clinicians. ${ }^{14}$ Thus, technologies that have the potential to provide innovative solutions to rehabilitation have failed to significantly impact the overall treatment plan.

Modern technological innovations can reduce barriers while modernizing physiotherapy at the same time. Using inexpensive and readily accessible technologies can help raise motivation and engagement levels without adding significant costs to either the healthcare system or the patients themselves. ${ }^{15-17}$ For example, wearables (eg Apple watch, Fitbit) are correlated with a significant increase in motivation and decrease in sedentary behaviors, ${ }^{18,19}$ but these devices have yet to be implemented for adherence in physical therapy. Similarly, VR-based video games have been used in research and treatment to help patients who suffer from chronic neck pain, ${ }^{20-}$ ${ }^{22}$ as well as to detect accuracy of cervical kinematics movements for rehabilitation. ${ }^{23}$ Furthermore, using VR-based simulation in mobility rehabilitation after a stroke is associated with improved gait in individuals. ${ }^{24}$ Finally, gamification of rehabilitation through VR has been shown to distract and ease anxiety experienced with treatment regimens in burn victims. ${ }^{25}$ Video games, therefore, have facilitated significant increases in intrinsic motivation that can help individuals overcome barriers in physical therapy adherence.

Gamification can help change behavioural patterns and encourage positive habits in people, which remains a difficult challenge in activity-based interventions. Video games are innately engaging and fun but are often regarded by health professionals as novelties. Video games designed solely by healthcare professionals, however, often lack the ability to intrinsically motivate users. ${ }^{26}$ Effective game design must include interdisciplinary collaboration between healthcare professionals, video game designers, and engineers in order to create video games that are engaging and effective. ${ }^{27}$

\section{CONCLUSION}

Physiotherapy can have significant positive outcomes on treatment length, outcomes, success and the overall quality of life. ${ }^{28}$ However, clinicians and patients both face difficulties in engagement and motivation. Video games designed specifically for rehabilitation can help address both of these issues without adding significant costs and strain to the healthcare system. It is important that future rehabilitation games require significant contributions from healthcare professions in both the design phase and testing phases to ensure success in a clinical environment. In turn, healthcare professionals must include engaging and fun elements to intrinsically motivate users. The success of rehabilitation- based video games can only be achieved through interdisciplinary collaboration between videogame designers and healthcare professionals.

\section{REFERENCES}

1. Jack K, McLean SM, Moffett JK, et al. Barriers to treatment adherence in physiotherapy outpatient clinics: a systematic review. Man Ther. 2010;15:220-8.

2. Essery R, Geraghty AW, Kirby S, et al. Predictors of adherence to home-based physical therapies: a systematic review. Disabil Rehabil. 2017;39:519-34.

3. Jolly K, Taylor R, Lip GY, et al. The Birmingham Rehabilitation Uptake Maximisation Study (BRUM). Home-based compared with hospital-based cardiac rehabilitation in a multi-ethnic population: cost-effectiveness and patient adherence. Health Technol Assess. 2007;11:1-118.

4. Jackson L, Leclerc J, Erskine Y, et al. Getting the most out of cardiac rehabilitation: a review of referral and adherence predictors. Heart. 2005;91:104.

5. Picorelli AM, Pereira LS, Pereira DS, et al. Adherence to exercise programs for older people is influenced by program characteristics and personal factors: a systematic review. J Physiother. 2014;60:151-6.

6. Hill A-M, Hoffmann T, McPhail S, et al. Factors associated with older patients' engagement in exercise after hospital discharge. Arch Phys Med Rehabil. 2011;92:1395-403.

7. Teo W-P, Muthalib M, Yamin S, et al. Does a combination of virtual reality, neuromodulation and neuroimaging provide a comprehensive platform for neurorehabilitation? - A narrative review of the literature. Front Hum Neurosci. 2016;10:284.

8. Johnson D, Deterding S, Kuhn K-A, et al. Gamification for health and wellbeing: A systematic review of the literature. Internet Interv. 2016;6:89-106.

9. Sailer M, Hense JU, Mayr SK, et al. How gamification motivates: An experimental study of the effects of specific game design elements on psychological need satisfaction. Comput Human Behav. 2017;69:371-80.

10. Kolt GS, Brewer BW, Pizzari T, et al. The Sport Injury Rehabilitation Adherence Scale: a reliable scale for use in clinical physiotherapy. Physiotherapy. 2007;93:17-22.

11. Deslauriers S, Raymond M-H, Laliberté M, et al. Access to publicly funded outpatient physiotherapy services in Quebec: waiting lists and management strategies. Disabil Rehabil. 2017;39:2648-56. https://doi.org/10.1080/09638288. 2016.1238967

12. Lateef F. Simulation-based learning: Just like the real thing. J Emerg Trauma Shock. 2010;3:348-52.

13. Székely G, Satava RM. Virtual reality in medicine. Interview by Judy Jones. BMJ. 1999;319:1305

14. Hardy S, Göbel S. Adaptation model for indoor exergames. Int J Comput Sci Sport. 2012;11:73-85

15. Chen Y-P, Kang L-J, Chuang T-Y, et al. Use of virtual reality to improve upperextremity control in children with cerebral palsy: a single-subject design. Phys Ther. 2007;87:1441-57.

16. Colombo R, Pisano F, Mazzone A, et al. Design strategies to improve patient motivation during robot-aided rehabilitation. J Neuroeng Rehabil. 2007;4:3.

17. Morris LD, Louw QA, Crous LC. Feasibility and potential effect of a low-cost virtual reality system on reducing pain and anxiety in adult burn injury patients during physiotherapy in a developing country. Burns. 2010;36:659-64.

18. Smith-Dektor AH, Young SD. Marketing, technology, and medicine: recommendations on how to incorporate psychological principles into new technologies to promote healthy behaviors. J Consum Health Internet. 2014;18:253-9.

19. Stephenson A, McDonough SM, Murphy MH, et al. Using computer, mobile and wearable technology enhanced interventions to reduce sedentary behaviour: a systematic review and meta-analysis. Int J Behav Nutr Phys Act. 2017;14:105. 
20. Sarig Bahat H, Sprecher E, Sela I, et al. Neck motion kinematics: an inter-tester reliability study using an interactive neck VR assessment in asymptomatic individuals. Eur Spine J. 2016 Jul;25(7):2139-48. https://doi.org/10.1007/s00586-016-4388-5

21. Sarig Bahat H, Takasaki H, Chen X, et al. Cervical kinematic training with and without interactive VR training for chronic neck pain - a randomized clinical trial. Man Ther. 2015 Feb;20(1):68-78. https://doi.org/10.1016/j.math.2014.06.008

22. Sarig Bahat $\mathrm{H}$, Croft $\mathrm{K}$, Carter C, et al. Remote kinematic training for patients with chronic neck pain: a randomised controlled trial. Eur Spine J. 2017 June;27(6):1309-23. https://doi.org/10.1007/s00586-017-5323-0

23. Kristjansson E, Hardardottir L, Asmundardottir M, et al. A new clinical test for cervicocephalic kinesthetic sensibility: "the fly." Arch Phys Med Rehabil. 2004;85:490-5.

24. Mirelman A, Bonato P, Deutsch JE. Effects of training with a robot-virtual reality system compared with a robot alone on the gait of individuals after stroke. Stroke. 2009;40:169-74.

25. Sharar SR, Miller W, Teeley A, et al. Applications of virtual reality for pain management in burn-injured patients. Expert Rev Neurother. 2008;8:1667-74.

26. Shahan Salim ML. Combining healthcare doctrine with game design principles to create effective exergames. Heal Sci Inq. 2018; In Press.

27. Richards C, Thompson CW, Graham N. Beyond designing for motivation: the importance of context in gamification. Proceedings of the first ACM SIGCHI annual symposium on Computer-human interaction in play - CHI PLAY '14; 2014 Oct 19-21; Toronto, Canada. New York: ACM Press; c2014. 217-26

28. Malmros B, Mortensen L, Jensen MB, et al. Positive effects of physiotherapy on chronic pain and performance in osteoporosis. Osteoporos Int. 1998;8:215-21 